\title{
The Relationship of Lymphocyte to High-Density Lipoprotein Ratio with Pulmonary Function in COPD
}

This article was published in the following Dove Press journal: International Journal of Chronic Obstructive Pulmonary Disease

\author{
Yiben Huang ${ }^{1} *$ \\ Bingqian Jiang ${ }^{1,2, *}$ \\ Xiaqi Miao ${ }^{1,2, *}$ \\ Jiedong $\mathrm{Ma}^{1,2}$ \\ Jianing Wang ${ }^{1,2}$ \\ Keke Ding ${ }^{1,2}$ \\ Xianjing Chen' \\ Qiaoming $\mathrm{Hu}^{1,2}$ \\ Fangyi $\mathrm{Fu}^{1,2}$ \\ Tian Zeng ${ }^{1,2}$ \\ Jingyu $\mathrm{Hu}^{1,2}$ \\ Binbin $\mathrm{Hu}^{1,2}$ \\ Dehao Yang ${ }^{3}$ \\ Xiaodiao Zhang (D)
}

'Department of Respiratory Medicine, The Third Affiliated Hospital of Wenzhou Medical University, Wenzhou, People's Republic of China; ${ }^{2}$ School of the First Clinical Medical Sciences, Wenzhou Medical University, Wenzhou, People's Republic of China; ${ }^{3}$ Department of Neurology, The Second Affiliated Hospital, Zhejiang University School of Medicine, Hangzhou, People's Republic of China

*These authors contributed equally to this work

Correspondence: Dehao Yang Department of Neurology, The Second Affiliated Hospital, Zhejiang University School of Medicine, Hangzhou 310009 Zhejiang, People's Republic of China

Tel +8613566209652

Email dehao_yang@zju.edu.cn

Xiaodiao Zhang

Department of Respiratory Medicine, The Third Affiliated Hospital of Wenzhou Medical University, No. 108 Wansong

Road, Wenzhou 325000 Zhejiang,

People's Republic of China

Tel +86-577-65866223

Fax +86-577-65866586

Email xiaodiao_zhang@।26.com
Purpose: This study aimed to explore the relation between lymphocyte to high-density lipoprotein ratio (LHR) and pulmonary function of chronic obstructive pulmonary disease (COPD) patients compared with neutrophil-lymphocyte ratio (NLR) and platelet-lymphocyte ratio (PLR).

Patients and Methods: In total, 154 participants $(n=77$ with COPD and $n=77$ without COPD) were recruited. LHR, NLR, PLR, lung function and other data were collected and compared. Pearson's correlation test and the receiver operating characteristics curves were used to compare the utility of LHR, NLR and PLR. Besides, univariate and multivariate logistic regression analyses were conducted.

Results: COPD patients with poorer lung function had a lower LHR level $(P<0.001)$. In low LHR group, more patients underwent greater airflow limitation than the other group $(P=$ 0.006). LHR positively correlated with forced expiratory volume in 1 second in percent of the predicted value $\left(\mathrm{FEV}_{1} \%\right)(r=0.333, P=0.003)$. At a cut-off value of 2.08 , the sensitivity and specificity of LHR in predicting $\mathrm{FEV}_{1} \%<50$ were $93.2 \%$ and $55.6 \%$, respectively, with an AUC of $0.770(P=0.001)$ better than NLR and PLR. Based on logistic regression analyses, it was proved that LHR was associated with decreased risk of $\mathrm{FEV}_{1}<50 \%$ predicted in COPD patients (odds ratio $=0.198,95 \%$ CI: $0.048-0.811, P=0.024$ ).

Conclusion: In contrast with NLR and PLR, LHR has higher accuracy for predicting pulmonary function in COPD; lower LHR level is independently associated with poorer pulmonary function.

Keywords: COPD, pulmonary function, lymphocytes, high-density lipoprotein

\section{Introduction}

Chronic obstructive pulmonary disease (COPD), a common chronic airway inflammatory disease characterized by persistent respiratory symptoms and airflow limitation, causes heavy social and economic burden owing to its high prevalence and relevant disability rate and mortality. ${ }^{1,2}$ Chronic inflammation predominantly affects the lung parenchyma and peripheral airways and results in largely irreversible and progressive airflow limitation in COPD. ${ }^{3}$ Inflammation is a complex set of interactions including neutrophils and lymphocytes. Acanfora ${ }^{4}$ observed a negative correlation between relative lymphocyte counts and mortality in elderly patients with severe COPD. It was reported that the neutrophil-lymphocyte ratio (NLR) was a favorable marker of systemic inflammation, acute exacerbation of COPD and mortality. ${ }^{5,6}$ Moreover, the platelet-lymphocyte ratio (PLR) may be a valuable 
index of ongoing inflammation and the disease severity of COPD. ${ }^{7}$ Nevertheless, Günay ${ }^{8}$ found no significant differences of NLR among I to IV groups divided according to Global Initiative for Chronic Obstructive Lung Disease (GOLD) and no studies reported the relationship of PLR and GOLD, which imply that a more credible biomarker of pulmonary function needs to be put forward. In addition, serum high-density lipoprotein (HDL) level might associate with COPD. A previous study showed that COPD individuals had a lower level of HDL than healthy controls. ${ }^{9}$ However, Park ${ }^{10}$ showed HDL-C levels were elevated in cohort with COPD compared to a reference population without COPD, but it was demonstrated that as the setting of emphysema being established, HDL might have dysfunctional properties and lose protective effect. ${ }^{11}$ Furthermore, lymphocyte to HDL ratio (LHR), representing both the lymphocyte counts and serum HDL levels, was advocated as a new indicator of inflammation and metabolic syndrome (MetS). ${ }^{12}$ However, none of the studies has investigated the potential value of LHR to serve as a biomarker of COPD, despite the fact that COPD is a chronic inflammatory disease.

Therefore, the aim of this cross-sectional study is to explore the relation between the LHR and the pulmonary function of COPD patients and compare the indicative role of LHR on COPD patients' pulmonary function with NLR and PLR.

\section{Patients and Methods}

\section{Study Population}

Between February 2018 and February 2019, we performed a cross-sectional research on COPD patients. One hundred and thirty-four subjects diagnosed with COPD were enrolled from the respiratory ward of the Third Affiliated Hospital of Wenzhou Medical University, 23 of them with missing data ( 9 subjects without LHR and 14 subjects without $\mathrm{FEV}_{1} \%$ ), and 3 of them with outliers were excluded. The inclusion criteria and exclusion criteria of patients were as follows: Inclusion criteria: 1) age more than 40 years; 2 ) diagnosis of COPD with GOLD $\geq 2^{13}$ as defined in the GOLD guidelines ${ }^{1}$ with symptoms of dyspnea, chronic cough, sputum production or wheezing; ${ }^{14}$ Exclusion criteria: ${ }^{12}$ 1) malignant tumor $\left.(\mathrm{n}=3) ; 2\right)$ hepatic insufficiency $(\mathrm{n}=1)$ and renal insufficiency $(\mathrm{n}=4) ; 3)$ heart diseases $(n=13) ; 4)$ autoimmune diseases $(n=2) ; 5)$ other lung diseases $(n=8) ; 31$ subjects meeting the exclusion criteria were excluded. Finally, a total of 77 patients were selected. All subjects with COPD were stratified into severity grades of airflow limitation based on $\mathrm{FEV}_{1}$ as percent of predicted with cut-offs according to GOLD grades $1-4 .^{4,13,15}$ And 77 age- and sex- matched healthy controls were included as control group who met the same inclusion and exclusion criteria as the COPD patients, except for the diagnosis of COPD.

The study was approved by the Ethics Committee of the Third Affiliated Hospital of Wenzhou Medical University and the registration number of Ethics Committee was YJ20170015. The study was conducted in accordance with the Declaration of Helsinki. All subjects signed a written informed consent form.

\section{Data Collection}

Data on age, gender, body mass index (BMI), smoking status and duration of disease were collected by questionnaires. Blood samples were collected to analyze blood routine parameters, blood biochemistry and arterial blood gas. Computable parameters such as LHR, NLR, PLR of COPD patients and healthy controls were calculated. Forced expiratory volume in 1 second $\left(\mathrm{FEV}_{1}\right)$, forced vital capacity (FVC), $\mathrm{FEV}_{1} / \mathrm{FVC}, \quad \mathrm{FEV}_{1}$ in percent of the predicted value $\left(\mathrm{FEV}_{1} \%\right)$ of COPD patients were recorded as significant indicators of the severity of pulmonary function. What is more, we also collected scales of COPD patients, including St. George's Respiratory Questionnaire (SGRQ) ${ }^{16}$ and BODE (BMI, airway obstruction, dyspnoea, severe exacerbations) index (BODE stages 1, 2, 3 and 4 were defined by BODE $0-2,3-4,5-6$, and $7-10$ points, respectively). ${ }^{17}$

\section{Diagnostic Criteria of COPD}

Patients with COPD were categorized in severity grades 1-4 using spirometry (GOLD 1: $\mathrm{FEV}_{1} \geq 80 \%$ predicted; GOLD 2: $50 \% \leq \mathrm{FEV}_{1}<80 \%$ predicted; GOLD 3: $30 \% \leq$ $\mathrm{FEV}_{1}<50 \%$ predicted; GOLD 4: $\mathrm{FEV}_{1}<30 \%$ predicted). ${ }^{14}$

\section{Statistical Analysis}

All statistical analysis was performed using SPSS 25.0 (IBM Analytics). Continuous variables of normal distribution were presented as mean \pm standard deviation. The independent sample $t$-test and the Mann-Whitney $U$-test were used to compare the differences of clinical characteristics between COPD patients and healthy controls, low LHR group and high LHR group. The data of patients stratified on the basis of $\mathrm{FEV}_{1} \%$ were compared through 
one-way analysis of variance (ANOVA) with Bonferroni as post hoc test or Welch's test. Additionally, categorical variables were expressed as counts and percentages, and intergroup comparisons were analyzed through Chisquared $\left(\chi^{2}\right)$ test or Fisher's exact test. Relationships between LHR/NLR/PLR and $\mathrm{FEV}_{1} \%$ were evaluated by Pearson's correlation test, while relationship between LHR and BODE index was evaluated by Spearman correlation test. In order to estimate the value of the novel indicator LHR for predicting pulmonary function compared with NLR and PLR, the receiver operating characteristics (ROC) curves were plotted. Propensity score matching (PS matching) (1:1 matching, caliper 0.1) was used to select healthy controls and adjust for imbalance of age, gender and smoking status in COPD patients. We used univariate logistic regression analysis to identify the variables associated with poor pulmonary function. Furthermore, multivariate logistic regression analysis was used to explain the contribution of the LHR in pulmonary function, controlling for confounders with $P<0.1$ in univariate logistic regression. To make OR estimates more reasonable, LHR was standardized by z-score in logistic regression analyses. Two-sided $P$ values $<0.05$ were considered significant in the other analyses.

\section{Results}

\section{Baseline Characteristics of the Study Subjects}

As shown in Table S1, among the 154 research candidates, 77 were diagnosed as COPD while 77 were age-and sexmatched healthy controls. Our results manifested that, compared with healthy controls, COPD patients had a higher level of neutrophils $(P<0.001)$, NLR $(P<$ $0.001)$, PLR $(P<0.001)$ and a lower level of lymphocytes $(P<0.001)$, HDL $(P<0.001)$. Moreover, we also found that a novel marker LHR was statistically lower in COPD than healthy controls $(P=0.018)$.

To gain a deeper understanding of the relationships between the three indicators above-mentioned and COPD severity of airflow limitation, we categorized 77 patients into three groups according to $\mathrm{FEV}_{1} \%$ (T1, $\mathrm{FEV}_{1} \% \geq 50$, $\mathrm{n}=18 ; \mathrm{T} 2,30 \leq \mathrm{FEV}_{1} \%<50, \mathrm{n}=35 ; \mathrm{T} 3, \mathrm{FEV}_{1} \%<30, \mathrm{n}=$ 24). As shown in Table 1, the differences were insignificant among these three groups in terms of gender, smoking status, white blood cells, neutrophils, platelets, triglycerides, total cholesterol (TC), low-density lipoprotein (LDL), NLR and PLR. However, age, BMI, lymphocytes, especially the LHR descended gradually from $\mathrm{T} 1$ to $\mathrm{T} 3$ group as $\mathrm{FEV}_{1} \%$ decreased $(P=0.003, P=0.001, P=0.009, P<0.001$,

Table I Baseline Characteristics of COPD Patients According to FEV $\%$

\begin{tabular}{|c|c|c|c|c|}
\hline Clinical Characteristics & $\mathrm{FEV}_{1} \% \geq 50(\mathrm{n}=18)$ & $30 \leq$ FEV $_{1} \%<50(n=35)$ & FEV $_{1} \%<30(n=24)$ & $P$ value \\
\hline Age (years) & $73.39 \pm 10.47$ & $72.34 \pm 7.26$ & $66.54 \pm 6.01$ & 0.003 \\
\hline Male sex, n (\%) & $13(72.2)$ & $27(77.1)$ & $23(95.8)$ & 0.073 \\
\hline Smoking status & & & & 0.285 \\
\hline Never-smoker, n (\%) & $4(22.2)$ & II (3I.4) & $2(8.3)$ & \\
\hline Former smoker, n (\%) & $10(55.6)$ & $17(48.6)$ & $16(66.7)$ & \\
\hline Current smoker, n (\%) & $4(22.2)$ & $7(20.0)$ & $6(25.0)$ & \\
\hline BMI $\left(\mathrm{kg} / \mathrm{m}^{2}\right)$ & $23.19 \pm 3.32$ & $21.25 \pm 3.53$ & $19.09 \pm 3.02$ & 0.001 \\
\hline WBC $(\times 10 * 9 / L)$ & $7.23 \pm 2.22$ & $6.84 \pm 2.86$ & $7.22 \pm 2.72$ & 0.824 \\
\hline Lymphocytes $(\times 10 * 9 / \mathrm{L})$ & $1.93 \pm 0.64$ & $1.46 \pm 0.57$ & $1.43 \pm 0.46$ & 0.009 \\
\hline Neutrophils $(\times 10 * 9 / \mathrm{L})$ & $4.56 \pm 1.89$ & $4.73 \pm 2.76$ & $4.99 \pm 2.38$ & 0.849 \\
\hline Platelets $(\times 10 * 9 / \mathrm{L})$ & $258.28 \pm 86.87$ & $222.60 \pm 65.49$ & $214.33 \pm 62.10$ & 0.113 \\
\hline Triglycerides $(\mathrm{mmol} / \mathrm{L})$ & $1.36 \pm 0.79$ & $1.02 \pm 0.43$ & $0.90 \pm 0.36$ & 0.078 \\
\hline $\mathrm{TC}(\mathrm{mmol} / \mathrm{L})$ & $4.32 \pm 0.89$ & $4.29 \pm 0.89$ & $4.33 \pm 0.77$ & $0.98 I$ \\
\hline $\mathrm{HDL}(\mathrm{mmol} / \mathrm{L})$ & $1.01 \pm 0.28$ & $1.09 \pm 0.24$ & $1.22 \pm 0.24$ & 0.028 \\
\hline LDL (mmol/L) & $2.63 \pm 0.72$ & $2.63 \pm 0.79$ & $2.63 \pm 0.68$ & 1.000 \\
\hline NLR & $2.55 \pm 1.22$ & $4.20 \pm 4.22$ & $4.01 \pm 2.61$ & 0.204 \\
\hline PLR & $144.06 \pm 56.60$ & $179.95 \pm 93.77$ & $170.22 \pm 99.57$ & 0.380 \\
\hline LHR & $1.98 \pm 0.65$ & $1.40 \pm 0.58$ & $1.25 \pm 0.52$ & $<0.001$ \\
\hline
\end{tabular}

Notes: Data are presented as mean \pm SD unless indicated otherwise.

Abbreviations: COPD, chronic obstructive pulmonary disease; $\mathrm{FEV}, \%$, forced expiratory volume in I second in percent of the predicted value; $\mathrm{BMI}$, body mass index; WBC, white blood cells; TC, total cholesterol; HDL, high-density lipoprotein; LDL, low-density lipoprotein; NLR, neutrophil-lymphocyte ratio; PLR, platelet-lymphocyte ratio; LHR, lymphocyte to HDL ratio; SD, standard deviation. 
respectively). On the contrary, the HDL level was ascending $(P=0.028)$.

In order to further explore the clinical values of LHR, subjects with COPD were divided into two groups according to the LHR median. In comparison to high LHR group $($ LHR $\geq 1.42, \mathrm{n}=38)$, low LHR group $($ LHR $<1.42, \mathrm{n}=$ 39) had statistically lower $\mathrm{FEV}_{1}, \mathrm{FVC}, \mathrm{FEV}_{1} \%$, platelets, triglycerides and $\mathrm{pH}$ levels. Conversely, incidences of higher SGRQ scores (SGRQ $\geq 25$ ), severer air flow limitation and advanced BODE stage and partial pressure of carbon dioxide in arterial blood $\left(\mathrm{PaCO}_{2}\right)$ in low LHR group were observably increased. No other significant differences were found in the rest of the studied parameters. Complete data are described in Table 2.

\section{Comparisons of the LHR, NLR and PLR in COPD}

Among COPD patients, we discovered patients with $\mathrm{FEV}_{1}$ $\% \geq 50$ had a significantly higher level of LHR than patients with $30 \leq \mathrm{FEV}_{1} \%<50(P=0.002)$ or $\mathrm{FEV}_{1} \%<$ $30(P<0.001)$. However, there were no differences of NLR nor PLR among each category of pulmonary function (Figure 1A).

Pearson's correlation test illustrated that LHR was positively related to $\mathrm{FEV}_{1} \%(r=0.333, P=0.003)$ while NLR and PLR were not (Figure 1B). Afterwards, the ROC curve analysis was performed to evaluate the utility of LHR, NLR and PLR for predicting poor pulmonary function $\left(\mathrm{FEV}_{1} \%<\right.$ 50). The analysis showed that the area under the curve (AUC) values of NLR and PLR were 0.617 (95\% CI: 0.478-0.756, $P=0.137)$ and 0.575 (95\% CI: $0.425-0.725, P=$ 0.339 ). In contrast, at a cut-off value of 2.08 , the sensitivity and specificity of the LHR in predicting poor pulmonary function were $93.2 \%$ and $55.6 \%$, respectively, with an AUC of 0.770 (95\% CI: $0.643-0.898, P=0.001$ ) (Figure 1C). It suggested that when it came to the severity of airway obstruction in COPD, LHR was positively associated with pulmonary function and had a higher predictive value compared with NLR and PLR.

\section{Declined LHR Level is Related to Poor Pulmonary Function}

Corresponding to above-mentioned analyses, participants with low LHR level had statistically lower $\operatorname{FEV}_{1}(P=$ $0.030), \mathrm{FVC}(P=0.039)$ and notably lower $\mathrm{FEV}_{1} \%(P=$ 0.018) (Figure $2 \mathrm{~A}$ ). In low LHR group, $87.2 \%$ suffered from poor pulmonary function $\left(\mathrm{FEV}_{1} \%<50\right)$ while the
Table 2 Baseline Characteristics of COPD Patients According to LHR Median

\begin{tabular}{|c|c|c|c|}
\hline $\begin{array}{l}\text { Clinical } \\
\text { Characteristics }\end{array}$ & $\begin{array}{l}\text { LHR }<1.42 \\
(n=39)\end{array}$ & $\begin{array}{l}\text { LHR } \geq 1.42 \\
(n=38)\end{array}$ & $P$ value \\
\hline Age (years) & $70.10 \pm 7.18$ & $71.47 \pm 9.19$ & 0.467 \\
\hline Male sex, n (\%) & $34(87.2)$ & $29(76.3)$ & 0.217 \\
\hline Smoking status & & & 0.733 \\
\hline Never-smoker, n (\%) & $6(15.4)$ & $12(31.6)$ & \\
\hline Former smoker, n (\%) & $28(71.8)$ & $15(39.5)$ & \\
\hline Current smoker, n (\%) & $5(12.8)$ & II (28.9) & \\
\hline BMI $\left(\mathrm{kg} / \mathrm{m}^{2}\right)$ & $20.47 \pm 3.64$ & $21.60 \pm 3.55$ & 0.175 \\
\hline $\begin{array}{l}\text { Duration of disease } \geq 5 \\
\text { years, } n(\%)\end{array}$ & $23(59.0)$ & $21(55.3)$ & 0.742 \\
\hline $\mathrm{FEV}_{1}(\mathrm{~L})$ & $0.82 \pm 0.32$ & $1.02 \pm 0.45$ & 0.030 \\
\hline FVC (L) & $1.63 \pm 0.46$ & $1.91 \pm 0.71$ & 0.039 \\
\hline $\mathrm{FEV}_{1} \%$ & $34.58 \pm|4.2|$ & $42.22 \pm 13.44$ & 0.018 \\
\hline $\mathrm{FEV}_{1} / \mathrm{FVC}$ & $49.63 \pm 9.23$ & $52.62 \pm 8.18$ & 0.137 \\
\hline SGRQ $\geq 25, \mathrm{n}(\%)$ & $32(82.1)$ & $23(60.5)$ & 0.037 \\
\hline Degree of air flow & & & 0.006 \\
\hline limitation & & & \\
\hline $\mathrm{FEV}_{1} \% \geq 50, \mathrm{n}(\%)$ & $5(12.8)$ & $13(34.2)$ & \\
\hline $30 \leq \mathrm{FEV}_{1} \%<50$ & $17(43.6)$ & I8 (47.4) & \\
\hline n (\%) & & & \\
\hline $\mathrm{FEV}_{1} \%<30, \mathrm{n}(\%)$ & $17(43.6)$ & $7(18.4)$ & \\
\hline BODE stages & & & 0.042 \\
\hline BODE I, n (\%) & $8(20.5)$ & $13(34.2)$ & \\
\hline BODE 2, n (\%) & $13(33.3)$ & $17(44.7)$ & \\
\hline BODE 3, n (\%) & $12(30.8)$ & $4(10.5)$ & \\
\hline BODE 4, n (\%) & $6(15.4)$ & $4(10.5)$ & \\
\hline WBC $(\times 10 * 9 / L)$ & $6.94 \pm 2.97$ & $7.16 \pm 2.32$ & 0.717 \\
\hline Neutrophils $(\times 10 * 9 / L)$ & $5.10 \pm 2.81$ & $4.44 \pm 1.98$ & 0.237 \\
\hline $\operatorname{RBC}(\times 10 * 12 / L)$ & $4.33 \pm 0.42$ & $4.24 \pm 0.54$ & 0.377 \\
\hline Hemoglobin $(g / L)$ & $130.65 \pm$ & $129.86 \pm$ & 0.815 \\
\hline & 11.96 & 17.02 & \\
\hline Platelet $(\times 10 * 9 / \mathrm{L})$ & $209.79 \pm$ & $247.42 \pm$ & 0.020 \\
\hline & 58.93 & 78.09 & \\
\hline Albumin $(g / L)$ & $36.17 \pm 2.87$ & $36.61 \pm 3.32$ & 0.534 \\
\hline Creatinine $(\mu \mathrm{mol} / \mathrm{L})$ & $66.43 \pm 11.66$ & $69.18 \pm|3.3|$ & 0.338 \\
\hline Triglycerides (mmol/L) & $0.85 \pm 0.33$ & $1.28 \pm 0.62$ & $<0.001$ \\
\hline $\mathrm{TC}(\mathrm{mmol} / \mathrm{L})$ & $4.40 \pm 0.78$ & $4.22 \pm 0.90$ & 0.334 \\
\hline $\mathrm{LDL}(\mathrm{mmol} / \mathrm{L})$ & $2.66 \pm 0.74$ & $2.60 \pm 0.73$ & 0.730 \\
\hline $\mathrm{pH}$ value & $7.40 \pm 0.03$ & $7.41 \pm 0.02$ & 0.024 \\
\hline $\mathrm{PaO}_{2}(\mathrm{mmHg})$ & $71.52 \pm 14.78$ & $74.78 \pm 13.97$ & 0.324 \\
\hline $\mathrm{PaCO}_{2}(\mathrm{mmHg})$ & $47.00 \pm 8.62$ & $42.96 \pm 5.47$ & 0.017 \\
\hline $\mathrm{SpO}_{2}$ & $93.06 \pm 4.05$ & $94.34 \pm 2.76$ & 0.110 \\
\hline
\end{tabular}

Notes: Data are presented as mean \pm SD unless indicated otherwise. Abbreviations: COPD, chronic obstructive pulmonary disease; LHR, lymphocyte to highdensity lipoprotein ratio; $\mathrm{BMI}$, body mass index; $\mathrm{FEV}_{\mathrm{l}}$, forced expiratory volume in I second; $\mathrm{FVC}$, forced vital capacity; FEV $\%$, FEV, in percent of the predicted value; SGRQ, St. George's Respiratory Questionnaire; BODE, BMI, airway obstruction, dyspnoea, severe exacerbations; WBC, white blood cells; RBC, red blood cells; TC, total cholesterol; LDL, low-density lipoprotein; $\mathrm{PaO}_{2}$, partial pressure of oxygen in arterial blood; $\mathrm{PaCO}_{2}$, partial pressure of carbon dioxide in arterial blood; $\mathrm{SpO}_{2}$, oxygen saturation; $\mathrm{SD}$, standard deviation. 
A
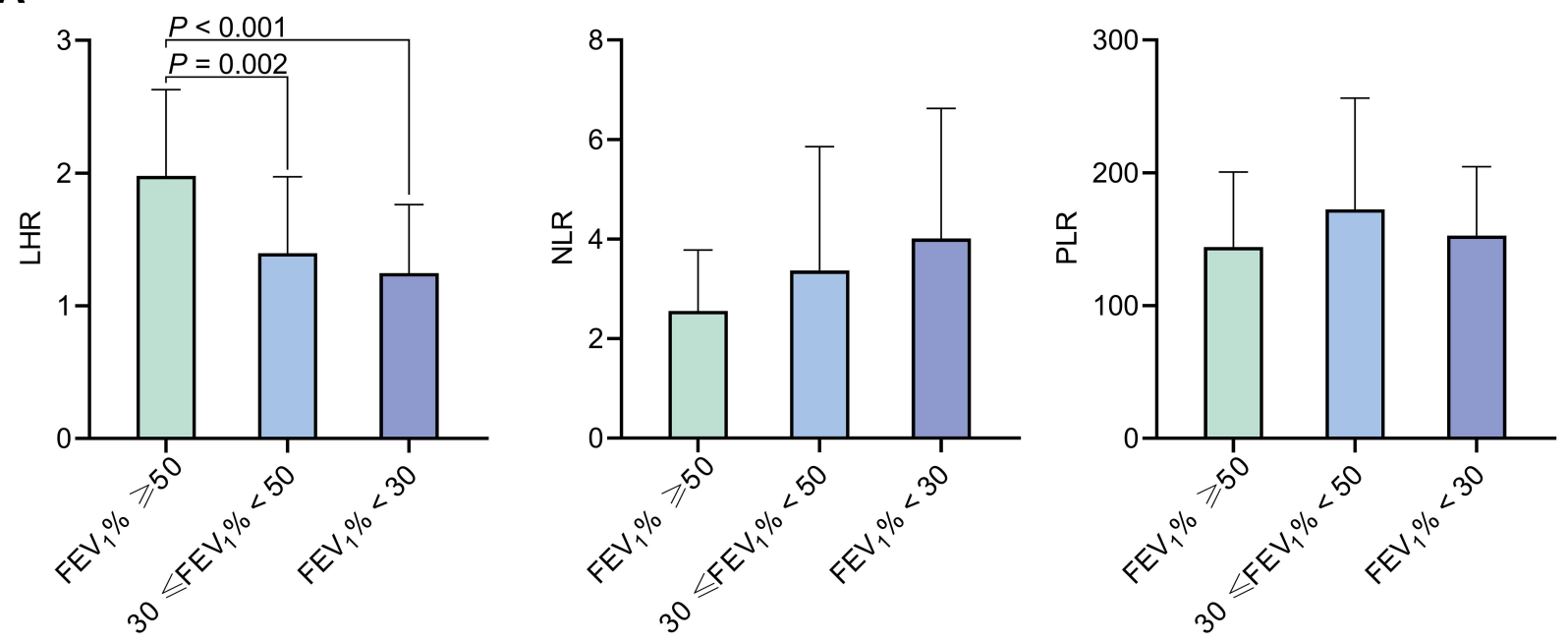

B
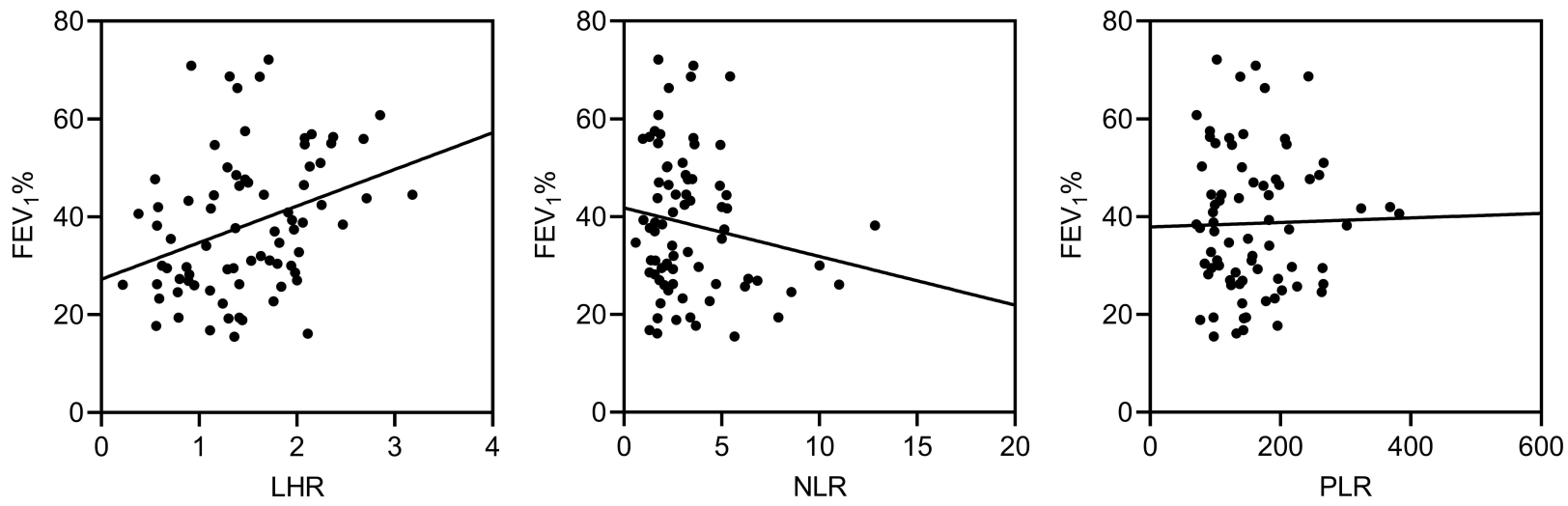

C

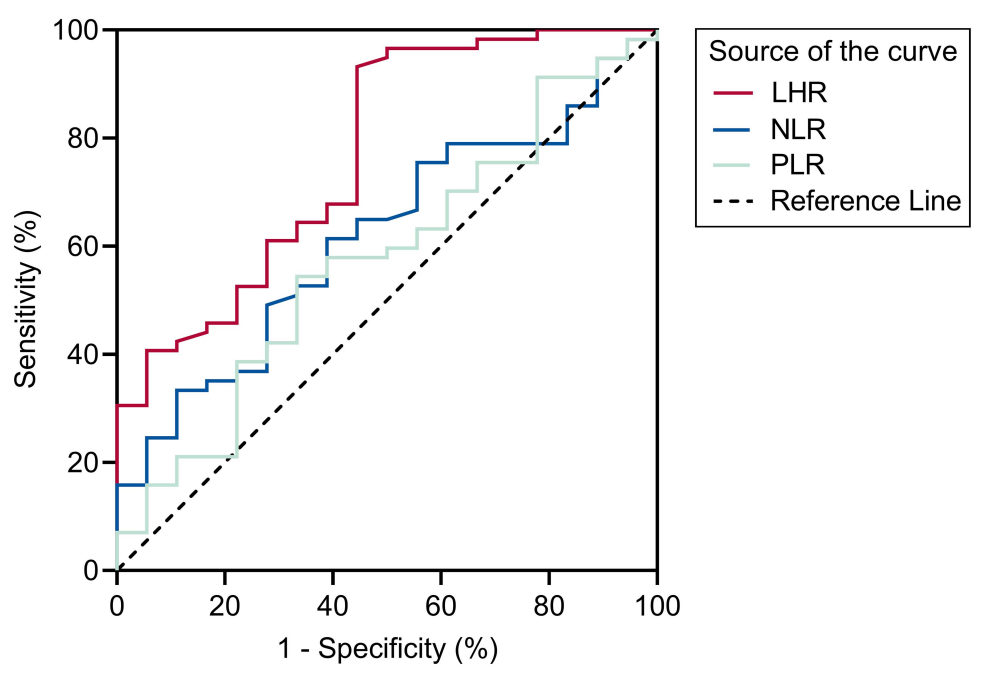

Figure I Comparisons of LHR, NLR and PLR in COPD patients. (A) LHR, NLR and PLR levels of COPD patients according to FEV 1 \%. (B) Correlations of the LHR, NLR and PLR with FEV $\%$. LHR, $r=0.333, P=0.003$; NLR, $r=-0.161, P=0.169$; and PLR, $r=0.022, P=0.849$. (C) ROC curves of the LHR, NLR and PLR for FEV $\%<50$ of COPD patients. The area under ROC curve (AUC) of LHR: $0.770,95 \% \mathrm{Cl}: 0.643-0.898, P=0.00 \mathrm{I}$; AUC of NLR: $0.617,95 \%$ Cl: $0.478-0.756, P=0.137$; and AUC of PLR: $0.575,95 \% \mathrm{Cl}: 0.425-0.725, P=0.339$. 

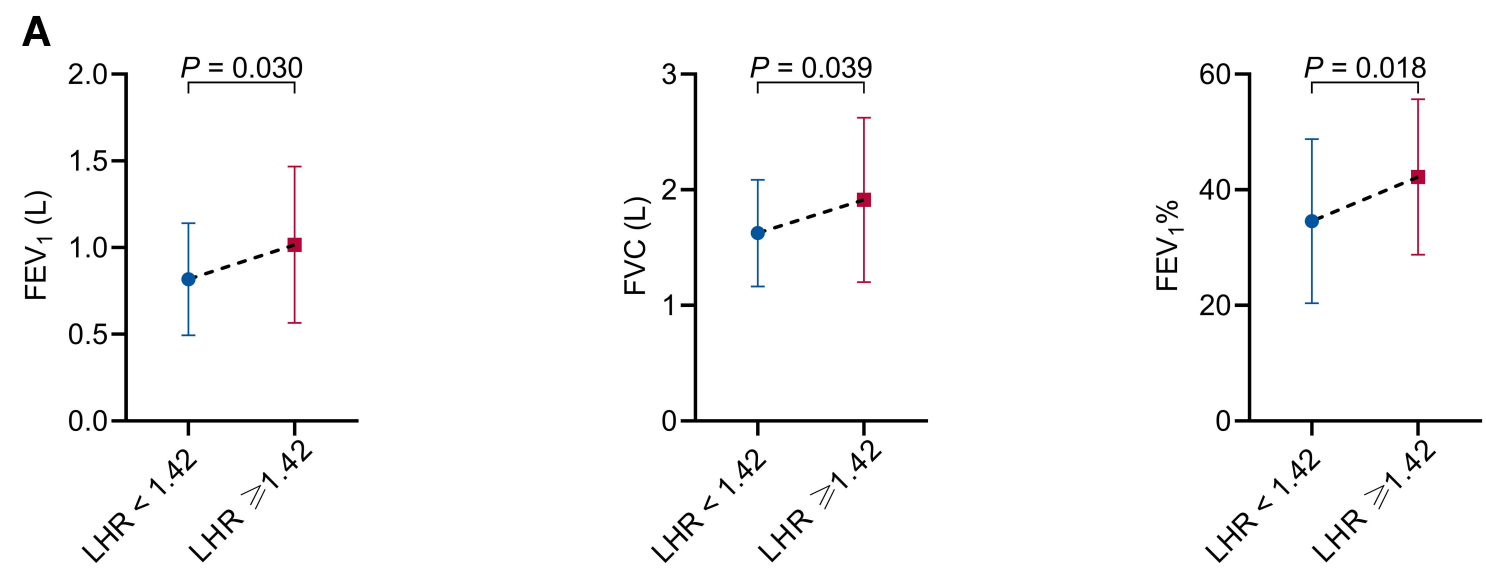

B

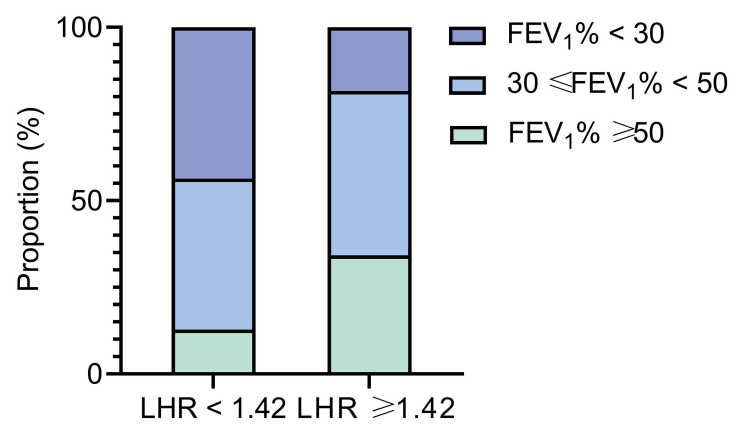

C

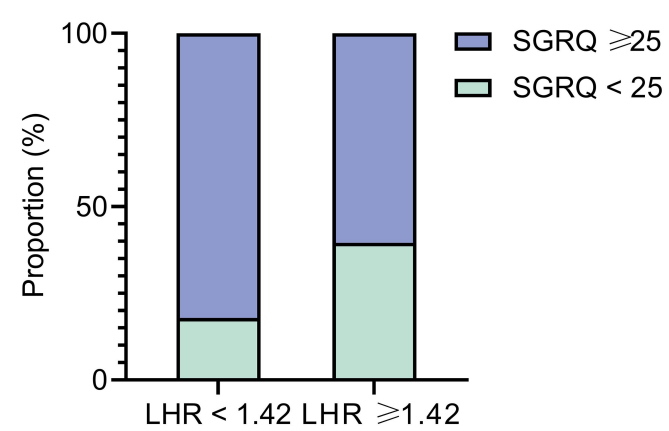

D

E
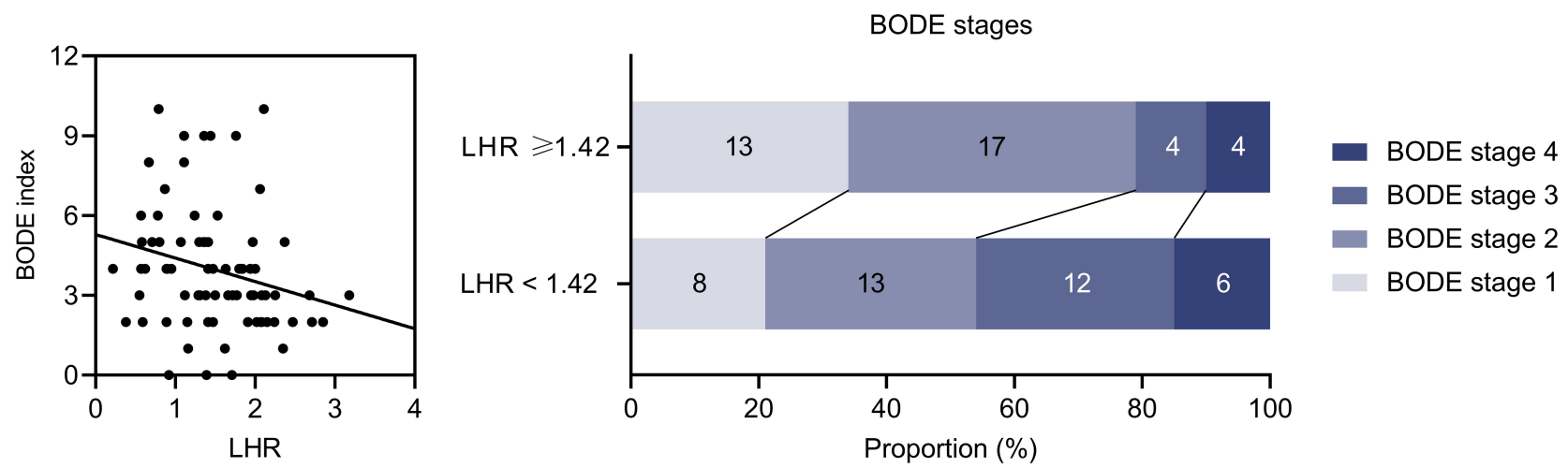

Figure 2 Comparisons of the condition of COPD patients according to the LHR median. (A) The FEV , FVC and FEV $\%$ level of COPD patients according to LHR. (B) The air flow limitation degree of COPD patients according to LHR. (C) The proportion of COPD patients with high SGRQ scores according to LHR. (D) Correlations of the BODE index with LHR, $\rho=-0.312, P=0.006$. (E) The BODE stages of COPD patients according to LHR.

proportion fell to $65.8 \%$ in high LHR group $(P=0.006$, Figure 2B). Besides, low LHR group comprised larger proportion of patients with SGRQ $\geq 25$ in contrast with high LHR group $(P=0.037$, Figure $2 C)$. Declined LHR level was also linked with advanced BODE index $(\rho=$ $-0.312, P=0.006$, Figure 2D; $\mathrm{P}=0.042$, Figure $2 \mathrm{E}$ ). These results implied that reduced LHR levels were related to disease severity and unfavorable outcomes of COPD.

Further, 33 subjects were selected after PS matching from low LHR group and high LHR group, respectively, to offset the impact of age, sex and smoking status in COPD patients when assessing the influence of LHR on parameters of pulmonary function. It demonstrated a higher 
level of $\mathrm{FEV}_{1}, \mathrm{FVC}, \mathrm{FEV}_{1} \%$ and $\mathrm{FEV}_{1} / \mathrm{FVC}(P=0.003$, $P=0.009, P=0.005, P=0.021$, respectively) in high LHR group of COPD patients (Table S2).

For the sake of determining the independent factors of pulmonary function, variables were subjected to univariate logistic regression analyses. Consequently, BMI $(P=0.006)$, $\mathrm{FEV}_{1} / \mathrm{FVC}(P<0.001), \mathrm{PaCO}_{2}(P=0.002)$, standardized LHR $(P=0.001)$ were observed to have significant correlations with $\mathrm{FEV}_{1} \%<50$ (Table 3). To control other potential confounding variables, multivariate logistic regression analyses were performed. In Model 1, nothing was adjusted (odds ratio $[\mathrm{OR}]=0.296,95 \% \mathrm{CI}: 0.145-0.603, P=$ 0.001). After adjusted for age, gender, smoking status, and duration of disease in Model 2, the linkage between standardized LHR and $\mathrm{FEV}_{1} \%<50$ remained significant (odds ratio $[\mathrm{OR}]=0.191,95 \%$ CI: $0.071-0.519, P=0.001)$. On the basis of Model 2, we also made adjustments for BMI, $\mathrm{FEV}_{1} / \mathrm{FVC}$, creatinine and $\mathrm{PaCO}_{2}$ in Model 3, and found that one unit increase in standardized LHR would reduce the risk of poor pulmonary function $\left(\mathrm{FEV}_{1} \%<50\right.$ ) by $80.2 \%$ (odds ratio $[\mathrm{OR}]=0.198,95 \%$ CI: $0.048-0.811, P=0.024)$. We substantially concluded that LHR is an independent marker of poor pulmonary function and increased LHR is associated with a reduced risk for COPD (Table 4).

\section{Discussion}

Our data indicated that lung function deteriorated with reduced LHR generated by low lymphocyte counts and high serum HDL level. It implied the potential correlation between lymphocyte counts or serum HDL level and lung function in COPD patients.

In the present study, lymphocyte counts were higher in COPD patients with $\mathrm{FEV}_{1} \%$ greater than 50 comparing with the others. Previous studies regarding lymphocytes and COPD are consistent with our findings. Autophagy has a fundamental role in the degradative pathway of lymphocytes, which plays critical roles in the development and pathogenesis of COPD inflammation. ${ }^{18,19}$ Lower lymphocyte counts were reported in patients with acute exacerbation of COPD than in the stable patients or the healthy controls. ${ }^{20}$ Acanfora ${ }^{4}$ identified that low relative lymphocyte counts were related to higher mortality in elderly severe COPD patients. Aging and COPD are associated with psychological stress, which leads to a significant increase in systemic cortisol production. Increased cortisol levels secreted by elderly COPD patients can give rise to gradual decrease in relative lymphocyte count. ${ }^{4}$ Furthermore, low lymphocyte counts
Table 3 Univariate Logistic Regression Analyses of Factors for $\mathrm{FEV}_{1} \%<50$

\begin{tabular}{|c|c|c|c|}
\hline Variables & OR & $95 \% \mathrm{Cl}$ & $P$ value \\
\hline Gender, female & 0.468 & $0.134-1.637$ & 0.235 \\
\hline \multicolumn{4}{|l|}{ Age (years) } \\
\hline$<60$ & 1.000 & & \\
\hline $60-69$ & 0.333 & $0.034-3.261$ & 0.345 \\
\hline $70-79$ & 0.406 & $0.044-3.758$ & 0.427 \\
\hline$\geq 80$ & 0.375 & $0.032-4.369$ & 0.434 \\
\hline \multicolumn{4}{|l|}{ Smoking status } \\
\hline Never-smoker & 1.000 & & \\
\hline Former smoker & 1.015 & $0.270-3.821$ & 0.982 \\
\hline Current smoker & 1.000 & $0.205-4.879$ & 1.000 \\
\hline BMI & 0.795 & $0.676-0.936$ & 0.006 \\
\hline $\begin{array}{l}\text { Duration of disease } \\
\geq 5 \text { years }\end{array}$ & 1.238 & $0.421-3.639$ & 0.698 \\
\hline $\mathrm{FEV}_{1} / \mathrm{FVC}$ & 0.842 & $0.77 \mid-0.920$ & $<0.001$ \\
\hline WBC & 0.967 & $0.796-1.175$ & 0.736 \\
\hline Neutrophils & 1.050 & $0.835-1.320$ & 0.676 \\
\hline $\mathrm{RBC}$ & 0.689 & $0.228-2.081$ & 0.509 \\
\hline Hemoglobin & 0.998 & $0.962-1.035$ & 0.906 \\
\hline Platelets & 0.994 & $0.986-1.002$ & 0.138 \\
\hline Albumin & 0.937 & $0.788-1.114$ & 0.463 \\
\hline Creatinine & 0.963 & $0.923-1.004$ & 0.076 \\
\hline Triglycerides & 0.405 & $0.127-1.298$ & 0.128 \\
\hline TC & 0.988 & $0.526-1.857$ & 0.971 \\
\hline LDL & 0.992 & $0.480-2.051$ & 0.983 \\
\hline $\mathrm{pH}$ value & $<0.001$ & $<0.001-143.197$ & 0.137 \\
\hline $\mathrm{PaO}_{2}$ & 0.987 & $0.953-1.023$ & 0.468 \\
\hline $\mathrm{PaCO}_{2}$ & 1.303 & $1.104-1.538$ & 0.002 \\
\hline $\mathrm{SpO}_{2}$ & 0.904 & $0.756-1.080$ & 0.266 \\
\hline NLR & 1.355 & $0.947-1.938$ & 0.097 \\
\hline PLR & 1.005 & $0.996-1.014$ & 0.279 \\
\hline LHR (standardized) & 0.296 & $0.145-0.603$ & 0.001 \\
\hline
\end{tabular}

Abbreviations: $\mathrm{FEV}, \%$, forced expiratory volume in I second in percent of the predicted value; $\mathrm{BMI}$, body mass index; $\mathrm{FEV}_{1}$, forced expiratory volume in I second; FVC, forced vital capacity; WBC, white blood cells; RBC, red blood cells; TC, total cholesterol; $\mathrm{LDL}$, low-density lipoprotein; $\mathrm{PaO}_{2}$, partial pressure of oxygen in arterial blood; $\mathrm{PaCO}_{2}$, partial pressure of carbon dioxide in arterial blood; $\mathrm{SpO}_{2}$, oxygen saturation; NLR, neutrophil-lymphocyte ratio; PLR, platelet-lymphocyte ratio; LHR, lymphocyte to high-density lipoprotein ratio.

might be put down to the impaired immunity. Since lymphocytes are crucial components of immune system, lymphopenia possesses a higher risk of respiratory tract infections, which are the most common causes of COPD exacerbations. ${ }^{1}$ Besides, malnutrition could also be responsible. Low lymphocyte counts qualify as a factor associated with nutritional risk ${ }^{21}$ whilst COPD severity is positively correlated with malnutrition. ${ }^{22}$ A vicious circle produced by neurohumoral activation and the immune 
Table 4 Adjusted Odds Ratio $(95 \% \mathrm{Cl})$ of Standardized LHR for $\mathrm{FEV}_{1} \%<50$

\begin{tabular}{|l|l|l|l|}
\hline & OR & $95 \% \mathbf{C l}$ & $P$ value \\
\hline Model I & 0.296 & $0.145-0.603$ & 0.001 \\
Model 2 & 0.191 & $0.071-0.519$ & 0.001 \\
Model 3 & 0.198 & $0.048-0.811$ & 0.024 \\
\hline
\end{tabular}

Notes: Model I is univariate analysis. Model 2 is adjusted by age; gender; smoking status; and duration of disease. Model 3 is adjusted by age; gender; smoking status; duration of disease; $\mathrm{BMI}$; $\mathrm{FEV} / \mathrm{FVC}$; creatinine; and $\mathrm{PaCO}_{2}$.

Abbreviations: LHR, lymphocyte to high-density lipoprotein ratio; $F E V_{1} \%$, forced expiratory volume in I second in percent of the predicted value; BMI, body mass index; $\mathrm{FEV}_{\mathrm{l}}$, forced expiratory volume in I second; FVC, forced vital capacity; $\mathrm{PaCO}_{2}$, partial pressure of carbon dioxide in arterial blood.

system demodulation increasing the secretion of cortisol may be to blame, ${ }^{4}$ as outlined in Figure 3 .

There are contradictory results about HDL and COPD. Qaisar $^{23}$ revealed that lower serum HDL level exacerbated the lung function in COPD patients, whereas elevated levels of HDL-C were found in cohort with COPD compared to a reference population without COPD ${ }^{10}$ Moreover, $\operatorname{Reed}^{24}$ stated that advanced COPD was associated with increased serum HDL level. Similarly, the inverse correlation between
HDL levels and pulmonary function has been reported recently. ${ }^{25}$ In our research, serum HDL levels were also elevated in patients with $\mathrm{FEV}_{1} \%$ less than 30 . This change might be partially attributable to the oral steroid use, which was an independent predictor of increased HDL-C in multivariate modeling. ${ }^{24}$ As shown in Figure 3, the underlying mechanism of HDL attenuating lung function in COPD patients involves the dual anti-inflammatory and proinflammatory nature of $\mathrm{HDL},{ }^{26} \mathrm{COPD}$ states associated with a chronic acute-phase response may trigger HDL to be dysfunctional and pro-inflammatory, thus further deteriorating disease status. Moreover, apolipoprotein $\mathrm{M}$ (apoM), as variation of a component of HDL, is elevated gradually with the COPD severity. ${ }^{27}$ ApoM and HDL are implicated in COPD pathogenesis, especially emphysema, via effecting ceramide, sphingosine-1-phosphate cellular levels and $\alpha_{1}$-antitrypsin. ${ }^{28}$

Consequently, we speculated that LHR, incorporating lymphocyte counts and serum HDL levels, was a feasible novel marker of the COPD airflow limitation severity. In the current study, LHR levels were related positively to $\mathrm{FEV}_{1} \%$ and could estimate lower $\mathrm{FEV}_{1} \%$ with a fair

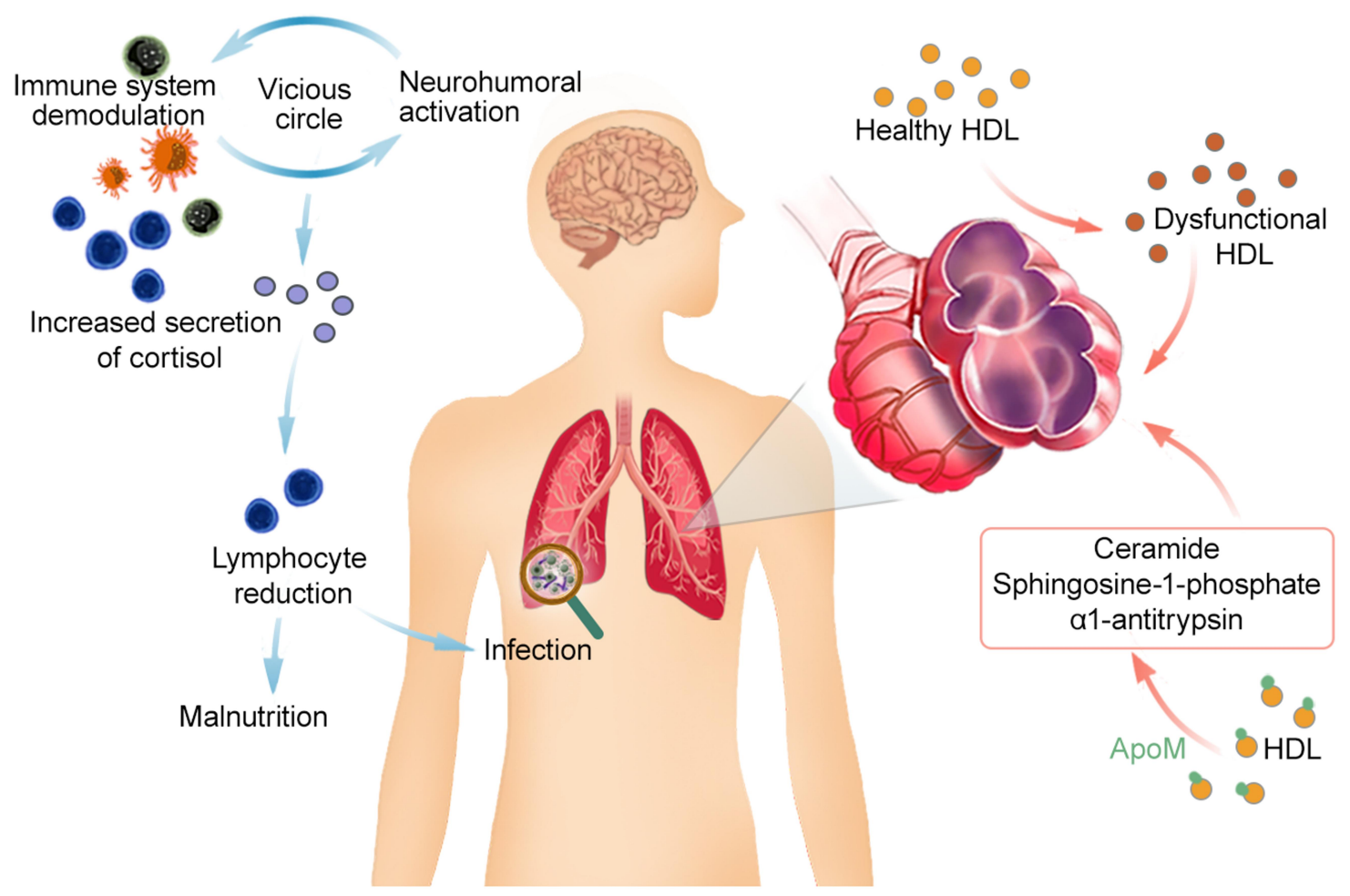

Figure 3 Role of the lymphocytes and HDL in COPD. A vicious circle produced by neurohumoral activation and immune system demodulation increases the secretion of cortisol, which causes the lymphocyte reduction. Lymphopenia possesses a higher risk of respiratory tract infections and malnutrition. As for HDL, COPD states trigger $\mathrm{HDL}$ to be dysfunctional and pro-inflammatory. And apolipoprotein M (apoM), as variations of a component of HDL, is implicated in COPD pathogenesis with HDL, especially emphysema, via effecting ceramide, sphingosine-I-phosphate cellular levels and $\alpha_{1}$-antitrypsin. Lymphocyte reduction and HDL conspire to deteriorate COPD status. 
accuracy. Nevertheless, we found it insignificant when it came to NLR and PLR. Parallel results were observed in several researches. Günay ${ }^{8}$ stated that NLR was significantly different between controls and COPD patients, but not between patients with stable and exacerbated COPD. $\mathrm{Lee}^{29}$ found no significant correlation of NLR with $\mathrm{FEV}_{1}$.

Furthermore, our data suggested that the LHR was associated with other outcome indicators of COPD. Among these COPD participants in our study, patients with elevated LHR levels showed lower BODE index and SGRQ scores, which represent the severity of COPD and health-related quality of life in COPD, respectively. ${ }^{30-32}$ The predictive role of LHR against lower $\mathrm{FEV}_{1} \%$ was substantiated via logistic regression analysis subsequently. Interestingly, some researchers have reported that current smokers had decreased HDL, which imply smoking status may play a potential role in the result of higher $\mathrm{FEV}_{1}$ in the LHR > 1.42 group in our study in conjunction with "healthy smoker" phenomenon. ${ }^{33-36}$ In our study, to weaken the influence of smoking status in COPD patients, we selected smoking-matched patients, and among them higher levels of lung function indexes were still found in high LHR group.

However, there are several limitations in our study. First, this preliminary study is a single-center cross-sectional survey limited on retrospective cohort. Second, the sample size is relatively small. Third, our work lacks exploring the cellular and molecular mechanisms of the impact of LHR on COPD. Prospective cohort studies with more participants recruited from multiple centers are needed to clarify this issue. And further researches need to be carried out to elucidate the role of LHR in COPD progression.

In conclusion, as a novel and promising marker, LHR can be calculated from the parameters of blood routine fast and conveniently. Lower levels of LHR were independently associated with poorer pulmonary function. It has predictive value for severer airway obstruction in COPD better than NLR and PLR. Our preliminary study could provide reference for clinicians to judge rapidly the pulmonary function of COPD patients. More attention should be attached to the LHR in clinical work.

\section{Abbreviations}

COPD, chronic obstructive pulmonary disease; LHR, lymphocyte to high-density lipoprotein ratio; NLR, neutrophil-lymphocyte ratio; PLR, platelet-lymphocyte ratio;
$\mathrm{FEV}_{1} \%$, forced expiratory volume in 1 second in percent of the predicted value; $\mathrm{FEV}_{1}$, forced expiratory volume in 1 second; BODE, body mass index, airway obstruction, dyspnoea, severe exacerbations; SGRQ, St. George's Respiratory Questionnaire; GOLD, Global Initiative for Chronic Obstructive Lung Disease; HDL, high-density lipoprotein; LDL, low-density lipoprotein; TC, total cholesterol; MetS, metabolic syndrome; BMI, body mass index; FVC, forced vital capacity; ROC, receiver operating characteristics; $\mathrm{PaCO}_{2}$, partial pressure of carbon dioxide in arterial blood; AUC, the area under the curve; $\mathrm{PaO}_{2}$, partial pressure of oxygen in arterial blood; $\mathrm{SpO}_{2}$, oxygen saturation; PS matching, propensity score matching; apoM, apolipoprotein M.

\section{Acknowledgments}

The authors thank all the participants in the study and the staff at the Third Affiliated Hospital of Wenzhou Medical University for their contribution in obtaining the data and assisting in the successful completion of the COPD study. The present study was supported by the Wenzhou Municipal Sci-Tech Bureau Program (Y20180365). Yiben Huang, Bingqian Jiang and Xiaqi Miao are co-first authors for this study.

\section{Author Contributions}

Yiben Huang: Conceptualization, Formal analysis, Methodology, Project administration, Supervision, Writing - original draft, Writing - review and editing. Bingqian Jiang: Data curation, Formal analysis, Investigation, Methodology, Project administration, Writing - original draft, Writing - review and editing. Xiaqi Miao: Data curation, Investigation, Writing - original draft, Writing - review and editing. Jiedong Ma, Jianing Wang, Keke Ding, Xianjing Chen, Qiaoming Hu, Fangyi $\mathrm{Fu}$, Tian Zeng, Jingyu Hu, Binbin Hu: Data curation, Investigation, Writing - review and editing. Dehao Yang, Xiaodiao Zhang: Conceptualization, Data curation, Investigation, Supervision, Writing - review and editing. All authors made substantial contributions to conception and design, acquisition of data, or analysis and interpretation of data; took part in drafting the article or revising it critically for important intellectual content; agreed to submit to the current journal; gave final approval of the version to be published; and agree to be accountable for all aspects of the work. 


\section{Disclosure}

The authors report no conflicts of interest.

\section{References}

1. Vogelmeier CF, Criner GJ, Martinez FJ, et al. Global strategy for the diagnosis, management, and prevention of chronic obstructive lung disease 2017 report. GOLD executive summary. Am J Respir Crit Care Med. 2017;195(5):557-582.

2. Soriano JB, Abajobir AA, Abate KH, Collaborators, G.B.D.C.R.D. Global, regional, and national deaths, prevalence, disability-adjusted life years, and years lived with disability for chronic obstructive pulmonary disease and asthma, 1990-2015: a systematic analysis for the Global Burden of Disease Study 2015. Lancet Respir Med. 2017;5(9):691-706. doi:10.1016/S2213-2600(17)30293-X

3. Barnes PJ. Inflammatory mechanisms in patients with chronic obstructive pulmonary disease. J Allergy Clin Immunol. 2016;138 (1):16-27. doi:10.1016/j.jaci.2016.05.011

4. Acanfora D, Scicchitano P, Carone M, et al. Relative lymphocyte count as an indicator of 3-year mortality in elderly people with severe COPD. BMC Pulm Med. 2018;18(1):116. doi:10.1186/s12890-0180685-6

5. Paliogiannis P, Fois AG, Sotgia S, et al. Neutrophil to lymphocyte ratio and clinical outcomes in COPD: recent evidence and future perspectives. Eur Respir Rev. 2018;27(147):147. doi:10.1183/ 16000617.0113-2017

6. Pascual-Gonzalez Y, López-Sánchez M, Dorca J, et al. Defining the role of neutrophil-to-lymphocyte ratio in COPD: a systematic literature review. Int J Chron Obstruct Pulmon Dis. 2018;13:3651-3662. doi: $10.2147 /$ COPD.S178068

7. Karadeniz G, Aktoğu S, Erer OF, et al. Predictive value of platelet-tolymphocyte ratio in exacerbation of chronic obstructive pulmonary disease. Biomark Med. 2016;10(7):701-710. doi:10.2217/bmm-20160046

8. Gunay E, Ulaşlı SS, Akar O, Ahsen A, Günay S, Koyuncu T, Ünlü M. Neutrophil-to-lymphocyte ratio in chronic obstructive pulmonary disease: a retrospective study. Inflammation. 2014;37 (2):374-380.

9. Gunay S, Sariaydin M, Acay A. New predictor of atherosclerosis in subjects with COPD: atherogenic indices. Respir Care. 2016;61 (11):1481-1487. doi:10.4187/respcare.04796

10. Park JH, Mun S, Choi DP, et al. Association between high-density lipoprotein cholesterol level and pulmonary function in healthy Korean adolescents: the JS high school study. BMC Pulm Med. 2017;17(1):190. doi:10.1186/s12890-017-0548-6

11. Gordon EM, Figueroa DM, Barochia AV, et al. High-density lipoproteins and apolipoprotein A-I: potential new players in the prevention and treatment of lung disease. Front Pharmacol. 2016;7:323. doi:10.3389/fphar.2016.00323

12. Chen H, Xiong C, Shao X, et al. Lymphocyte to high-density lipoprotein ratio as a new indicator of inflammation and metabolic syndrome. Diabetes Metab Syndr Obes. 2019;12:2117-2123. doi:10.2147/DMSO.S219363

13. Backman H, Eriksson B, Rönmark E, et al. Decreased prevalence of moderate to severe COPD over 15 years in northern Sweden. Respir Med. 2016;114:103-110.

14. Mirza S, Clay RD, Koslow MA, et al. COPD guidelines: a review of the 2018 GOLD report. Mayo Clin Proc. 2018;93(10):1488-1502. doi:10.1016/j.mayocp.2018.05.026

15. Viinanen A, Lassenius MI, Toppila I, et al. The burden of Chronic Obstructive Pulmonary Disease (COPD) in Finland: impact of disease severity and eosinophil count on healthcare resource utilization. Int J Chron Obstruct Pulmon Dis. 2019;14:2409-2421. doi:10.2147/ COPD.S222581
16. Jones PW, Quirk FH, Baveystock CM, et al. A self-complete measure of health status for chronic airflow limitation: the St. George's respiratory questionnaire. Am Rev Respir Dis. 1992;145 (6):1321-1327. doi:10.1164/ajrccm/145.6.1321

17. Celli BR, Cote CG, Marin JM, et al. The body-mass index, airflow obstruction, dyspnea, and exercise capacity index in chronic obstructive pulmonary disease. $N$ Engl J Med. 2004;350(10):1005-1012. doi:10.1056/NEJMoa021322

18. Wang Y, Xu J, Meng Y, et al. Role of inflammatory cells in airway remodeling in COPD. Int $J$ Chron Obstruct Pulmon Dis. 2018;13:3341-3348. doi:10.2147/COPD.S176122

19. Matsuzawa-Ishimoto Y, Hwang S, Cadwell K. Autophagy and inflammation. Аnпu Rev Immunol. 2018;36(1):73-101. doi:10.1146/ annurev-immunol-042617-053253

20. Sahin F, Koşar AF, Aslan AF, et al. Serum biomarkers in patients with stable and acute exacerbation of chronic obstructive pulmonary disease: a comparative study. J Med Biochem. 2019;38(4):503-511. doi:10.2478/jomb-2018-0050

21. Leandro-Merhi VA, Costa CL, Saragiotto L, et al. Nutritional indicators of malnutrition in hospitalized patients. Arq Gastroenterol. 2019;56(4):447-450. doi:10.1590/s0004-2803.201900000-74

22. Collins PF, Elia M, Kurukulaaratchy RJ, et al. The influence of deprivation on malnutrition risk in outpatients with chronic obstructive pulmonary disease (COPD). Clin Nutr. 2018;37(1):144-148. doi:10.1016/j.clnu.2016.11.005

23. Qaisar R, Karim A, Muhammad T. Circulating biomarkers of handgrip strength and lung function in chronic obstructive pulmonary disease. Int $J$ Chron Obstruct Pulmon Dis. 2020;15:311-321. doi:10.2147/COPD.S225765

24. Reed RM, Iacono A, DeFilippis A, et al. Advanced chronic obstructive pulmonary disease is associated with high levels of high-density lipoprotein cholesterol. J Heart Lung Transplant. 2011;30 (6):674-678. doi:10.1016/j.healun.2010.12.010

25. Huerta-Ramirez S, Paniagua-Pérez A, Castro-Serna D, LedesmaVelázquez A, Rubio-Guerra A, Vargas-Ayala G. Effect of the components of the metabolic syndrome on pulmonary function. The unexpected role of high-density lipoprotein cholesterol. Cir Cir. 2018;86(2):175-181.

26. Namiri-Kalantari R, Gao F, Chattopadhyay A, et al. The dual nature of HDL: anti-inflammatory and pro-inflammatory. Biofactors. 2016;7 (3):153-159. doi:10.1002/biof.1205

27. Li H, Liu Y, Wang L, et al. High apolipoprotein M serum levels correlate with chronic obstructive pulmonary disease. Lipids Health Dis. 2017;5(1):59. doi:10.1186/s12944-016-0228-1

28. Burkart KM, Manichaikul A, Wilk JB, et al. APOM and high-density lipoprotein cholesterol are associated with lung function and per cent emphysema. Eur Respir J. 2020;15(4):311-321. doi:10.1183/ 09031936.00147612

29. Lee SJ, Lee HR, Lee TW, et al. Usefulness of neutrophil to lymphocyte ratio in patients with chronic obstructive pulmonary disease: a prospective observational study. Korean J Intern Med. 2016;31 (5):891-898. doi:10.3904/kjim.2015.084

30. Camiciottoli G, Bigazzi F, Bartolucci M, et al. BODE-index, modified BODE-index and ADO-score in chronic obstructive pulmonary disease: relationship with COPD phenotypes and CT lung density changes. COPD. 2012;9(3):297-304. doi:10.3109/ 15412555.2012.661000

31. Donaria L, Mesquita R, Martinez L, et al. Relationship between sniff nasal inspiratory pressure and BODE index in patients with COPD. Lung. 2014;192(6):897-903. doi:10.1007/s00408-014-9649-7

32. Folch Ayora A, Macia-Soler L, Orts-Cortés MI, et al. Comparative analysis of the psychometric parameters of two quality-of-life questionnaires, the SGRQ and CAT, in the assessment of patients with COPD exacerbations during hospitalization: a multicenter study. Chron Respir Dis. 2018;15(4):374-383. doi:10.1177/ 1479972318761645 
33. Nakamura K, Barzi F, Huxley R, et al. Does cigarette smoking exacerbate the effect of total cholesterol and high-density lipoprotein cholesterol on the risk of cardiovascular diseases? Heart. 2009;95 (11):909-916. doi:10.1136/hrt.2008.147066

34. Gepner AD, Piper ME, Johnson HM, et al. Effects of smoking and smoking cessation on lipids and lipoproteins: outcomes from a randomized clinical trial. Am Heart J. 2011;161(1):145-151. doi:10.1016/j.ahj.2010.09.023
35. He B-M, Zhao S-P, Peng Z-Y. Effects of cigarette smoking on HDL quantity and function: implications for atherosclerosis. $J$ Cell Biochem. 2013;114(11):2431-2436. doi:10.1002/jcb.24581

36. Becklake MR, Lalloo U. The 'healthy smoker': a phenomenon of health selection? Respiration. 1990;57(3):137-144. doi:10.1159/ 000195837

International Journal of Chronic Obstructive Pulmonary Disease

\section{Publish your work in this journal}

The International Journal of COPD is an international, peer-reviewed journal of therapeutics and pharmacology focusing on concise rapid reporting of clinical studies and reviews in COPD. Special focus is given to the pathophysiological processes underlying the disease, intervention programs, patient focused education, and self management protocols. This journal is indexed on PubMed Central, MedLine and CAS. The manuscript management system is completely online and includes a very quick and fair peer-review system, which is all easy to use. Visit http://www.dovepress.com/testimonials.php to read real quotes from published authors.

Submit your manuscript here: https://www.dovepress.com/international-journal-of-chronic-obstructive-pulmonary-disease-journal 